\title{
ALMA observations of molecular absorption in four directions toward the Galactic bulge
}

\author{
H. Liszt ${ }^{1}$ and M. Gerin ${ }^{2}$ \\ 1 National Radio Astronomy Observatory, 520 Edgemont Road, Charlottesville, VA 22903, USA \\ e-mail: hliszt@nrao.edu \\ 2 LERMA, Observatoire de Paris, PSL Research University, CNRS, Sorbonne Universités, UPMC Univ. Paris 06, École Normale \\ Supérieure, 75005 Paris, France \\ e-mail: maryvonne.gerin@ens.fr
}

Received 22 September 2017 / Accepted 5 November 2017

\section{ABSTRACT}

\begin{abstract}
Context. Alma Cycle 3 observations serendipitously showed strong absorption from diffuse molecular gas in the Galactic bulge at $-200 \mathrm{~km} \mathrm{~s}^{-1}<v<-140 \mathrm{~km} \mathrm{~s}^{-1}$ toward the compact extragalactic continuum source $\mathrm{J} 1744-3116$ at $(l, b)=-2.13^{\circ},-1.00^{\circ}$.

Aims. We aimed to test whether molecular gas in the bulge could also be detected toward the three other, sufficiently strong mm-wave continuum sources seen toward the bulge at $|b|<3^{\circ}$.

Methods. We took absorption profiles of $\mathrm{HCO}^{+}(1-0), \mathrm{HCN}(1-0), \mathrm{C}_{2} \mathrm{H}(1-0), \mathrm{CS}(2-1)$ and $\mathrm{H}^{13} \mathrm{CO}^{+}(1-0)$ in ALMA Cycle 4 toward J1713-3418, J1717-3341, J1733-3722 and J1744-3116.

Results. Strong molecular absorption from disk gas at $|v| \lesssim 30 \mathrm{~km} \mathrm{~s}^{-1}$ was detected in all directions, and absorption from the $3 \mathrm{kpc}$ arm was newly detected toward J1717 and J1744. However, only the sightline toward J1744 is dominated by molecular gas overall and no other sightlines showed molecular absorption from gas deep inside the bulge. No molecular absorption was detected toward J1717 where $\mathrm{HI}$ emission from the bulge was previously known. As observed in $\mathrm{HCO}^{+}, \mathrm{HCN}, \mathrm{C}_{2} \mathrm{H}$ and $\mathrm{CS}$, the bulge gas toward $\mathrm{J} 1744$ at $v<-135 \mathrm{~km} \mathrm{~s}^{-1}$ has chemistry and kinematics like that seen near the Sun and in the Milky Way disk generally. We measured isotopologic ratios $N\left(\mathrm{HCO}^{+}\right) / N\left(\mathrm{H}^{13} \mathrm{CO}^{+}\right)>51(3 \sigma)$ for the bulge gas toward $\mathrm{J} 1744$ and $58 \pm 9$ and $64 \pm 4$ for the disk gas toward $\mathrm{J} 1717$ and J1744, respectively, all well above the value of 20-25 typical of the central molecular zone.

Conclusions. The kinematics and chemistry of the bulge gas observed toward J1744 more nearly resemble that of gas in the Milky Way disk than in the central molecular zone.
\end{abstract}

Key words. ISM: molecules - ISM: clouds - ISM: abundances

\section{Introduction}

In a recent paper (Gerin \& Liszt 2017) we discussed the serendipitous discovery of strong molecular absorption at $-210 \mathrm{~km} \mathrm{~s}^{-1} \leq v \leq-135 \mathrm{~km} \mathrm{~s}^{-1}$ toward the compact extragalactic continuum source J1744-3116 (aka B1741-312) at $l, b=-2.13^{\circ},-1.00^{\circ}$, along a line of sight passing through the inner portions of the Galactic bulge outside the central molecular zone (CMZ; Morris \& Serabyn 1996). We extracted the run of IR $K$-band extinction with distance modulus from the 3D extinction maps of Schultheis et al. (2014) and showed that the $K$ band extinction in the bulge implied the existence of unseen gas, given the weakness of $\mathrm{HI}$ emission at high negative velocities characteristic of the bulge. The $\mathrm{H}_{2}$ column density inferred from $\mathrm{HCO}^{+}$absorption matched the amount of gas lacking in $\mathrm{HI}$.

As we discussed, the quantity of molecular gas in the bulge outside the $\mathrm{CMZ}$, at $1.2^{\circ} \lesssim|l| \lesssim 10^{\circ}$ or galactocentric radius $180 \mathrm{pc} \lesssim R \lesssim 1500 \mathrm{kpc}$ at the IAU standard distance $R_{0}=$ $8.5 \mathrm{kpc}$, is rather uncertain. The total mass of $\mathrm{HI}$ in the bulge is comparable to the mass of $\mathrm{H}_{2}$ in the $\mathrm{CMZ}, M(\mathrm{HI}) \approx 3 \times 10^{7} M_{\odot}$ (Burton \& Liszt 1978), raising the possibility that the mass of molecular gas in the bulge is yet larger.

Mm-wave absorption toward J1744 from a wider range of species was subsequently discussed by Riquelme et al. (2018) who pointed out some of the same similarities between the chemical composition of the bulge and disk gases that are noted here. In the present work we re-observed molecular absorption toward J1744 with more complete spectral coverage than in our earlier discussion, at much higher spectral resolution than in our earlier work or in Riquelme et al. (2018). We also searched for similar absorption toward the few (three) known, strong mmwave continuum sources lying toward the bulge within $3^{\circ}$ of the Galactic plane. All of the newly-observed sightlines lie much further from the Galactic center than J1744, and none of them show molecular absorption at velocities characteristic of gas deep inside the bulge: we did find two weak features arising in the $3 \mathrm{kpc}$ arm (Bania 1977, 1980; Dame \& Thaddeus 2008).

The plan of this work is as follows: in Sect. 2 we describe the new and existing observational material that is discussed. In Sect. 3 we briefly summarize some properties of the observed sightlines and the disk gas that is present along them. In Sect. 4 we present new observational results leading to a better characterization of the bulge gas toward J1744: it has a chemistry and cloud structure comparable to that seen in $\mathrm{HCO}^{+}, \mathrm{C}_{2} \mathrm{H}, \mathrm{HCN}$ and CS in diffuse molecular gas near the Sun and, unlike material in the $\mathrm{CMZ}$, is not enhanced in ${ }^{13} \mathrm{C}$. Section 5 is a summary and discussion.

\section{Observations and data reduction}

\subsection{New ALMA absorption measurements}

We observed the $J=1-0$ lines of $\mathrm{HCO}^{+}, \mathrm{H}^{13} \mathrm{CO}^{+}, \mathrm{HCN}$ and $\mathrm{C}_{2} \mathrm{H}$, and the $J=2-1$ line of $\mathrm{CS} J=2-1$ in absorption 

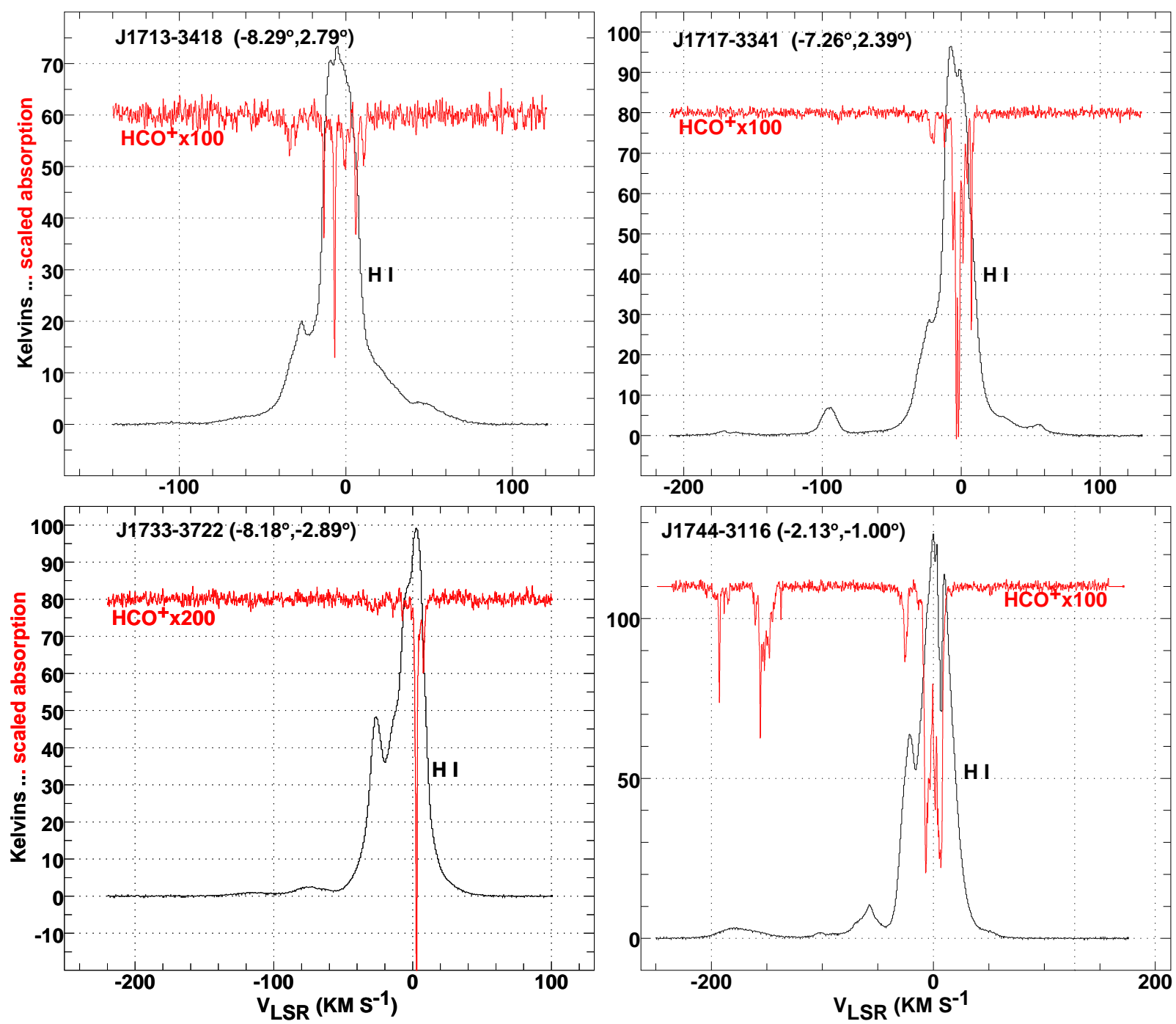

Fig. 1. GASS H I and ALMA $J=1-0 \mathrm{HCO}^{+}$profiles for the four sources observed here with ALMA.

Table 1. Observed and derived quantities.

\begin{tabular}{|c|c|c|c|c|c|c|c|c|c|c|}
\hline Source & $\begin{array}{l}l, b \\
\mathrm{o}, \mathrm{o} \\
\end{array}$ & $\begin{array}{l}S_{v} \\
\mathrm{Jy}\end{array}$ & $\begin{array}{l}R_{<}{ }^{a} \\
\mathrm{kpc}\end{array}$ & $\begin{array}{l}A_{K}^{b} \\
\mathrm{mag} \\
\end{array}$ & $\begin{array}{c}N(\mathrm{HI})_{0}{ }^{c} \\
10^{22} \mathrm{~cm}^{-2}\end{array}$ & $\begin{array}{c}(\mathrm{H} \mathrm{I})_{\tau}{ }^{d} \\
10^{22} \mathrm{~cm}^{-2}\end{array}$ & $\begin{array}{c}2 N\left(\mathrm{H}_{2}\right)^{e} \\
10^{22} \mathrm{~cm}^{-2}\end{array}$ & $\begin{array}{c}N(\mathrm{H}) \\
10^{22} \mathrm{~cm}^{-2}\end{array}$ & $\overline{f_{\mathrm{H}_{2}}{ }^{f}}$ & $\begin{array}{c}N(\mathrm{H}) / A_{K} \\
10^{22} \mathrm{~cm}^{-2} \mathrm{mag}^{-1}\end{array}$ \\
\hline J1713-3418 & $351.705,+2.787$ & 0.93 & 1.23 & 0.40 & 0.43 & $(0.64)^{g}$ & 0.18 & $(0.82)$ & $(0.22)$ & $>1.53$ \\
\hline J1717-3341 & $352.733,+2.391$ & 0.49 & 1.08 & 0.66 & 0.54 & 0.78 & 0.53 & 1.31 & 0.40 & 1.98 \\
\hline J1733-3722 & $351.818,-2.889$ & 0.60 & 1.21 & 0.60 & 0.56 & (1.13) & 0.11 & $(1.24)$ & $(0.09)$ & $>1.12$ \\
\hline J1744-3116 & $357.863,-0.997$ & 0.33 & 0.32 & 1.46 & 0.90 & 1.20 & 1.93 & 3.13 & 0.62 & 2.14 \\
\hline
\end{tabular}

Notes. ${ }^{(a)} R_{<}=R_{0} \sin (|l|), R_{0}=8500$ pc. ${ }^{(b)}$ Values from Schultheis et al. (2014); $E(B-V) / A_{K}=3.09$ (Schlafly \& Finkbeiner 2011). (c) $N(\mathrm{HI})_{0}=1.823 \times 10^{18} \mathrm{~cm}^{-2} \int T_{\mathrm{B}}(v) \mathrm{d} v{ }^{(d)} N(\mathrm{HI})_{\tau}$ from Eq. $(1) .{ }^{(e)} N\left(\mathrm{H}_{2}\right)=N\left(\mathrm{HCO}^{+}\right) / 3 \times 10^{-9}, N\left(\mathrm{HCO}^{+}\right)=1.10 \times 10^{12} \mathrm{~cm}^{-2} \int \tau_{\mathrm{HCO}^{+}} \mathrm{d} v$. (f) $f_{\mathrm{H}_{2}}=2 N\left(\mathrm{H}_{2}\right) /\left(N(\mathrm{HI})+2 N\left(\mathrm{H}_{2}\right)\right)$. ${ }^{(g)}$ Parenthesized quantities assume $N(\mathrm{H}) / A_{K}=2.06 \times 10^{22} \mathrm{~cm}^{-2} \mathrm{mag}^{-1}$, the average for J1717 and J1744.

toward the four continuum sources listed in Table 1. The work was conducted under ALMA Cycle 4 project 2016.1.00132.S whose pipeline data products were delivered in 2017 February. The spectra discussed here were extracted from the continuumsubtracted pipeline-processed data cubes at the pixel of peak continuum flux in the continuum map made from each spectral window, and divided by the continuum flux in the continuum map at that pixel. Fluxes at $89.2 \mathrm{GHz}$ ranged from $0.33 \mathrm{Jy}$ for $\mathrm{J} 1713$ to $0.93 \mathrm{Jy}$ for $\mathrm{J} 1733$. Each spectrum consisted of 1919 semi-independent channels spaced $61.13 \mathrm{kHz}$ corresponding to $0.205 \mathrm{~km} \mathrm{~s}^{-1}$ at the $89.189 \mathrm{GHz}$ rest frequency of $\mathrm{HCO}^{+}$ or $0.187 \mathrm{~km} \mathrm{~s}^{-1}$ for $\mathrm{CS}(2-1)$ at $97.981 \mathrm{GHz}$ : the channel spacing is half the spectral resolution. Velocities presented with the spectra are taken with respect to the kinematic definition of the Local Standard of Rest.

\subsection{Other data}

In Fig. 1 we compare our $\mathrm{HCO}^{+}$spectra with Galactic All-Sky Survey (GASS) III $\lambda 21 \mathrm{~cm}$ HI emission spectra (Kalberla \& Haud 2015). For the sources J1717 and J1744 we use the H I absorption profiles of Dickey et al. (1983) to derive 

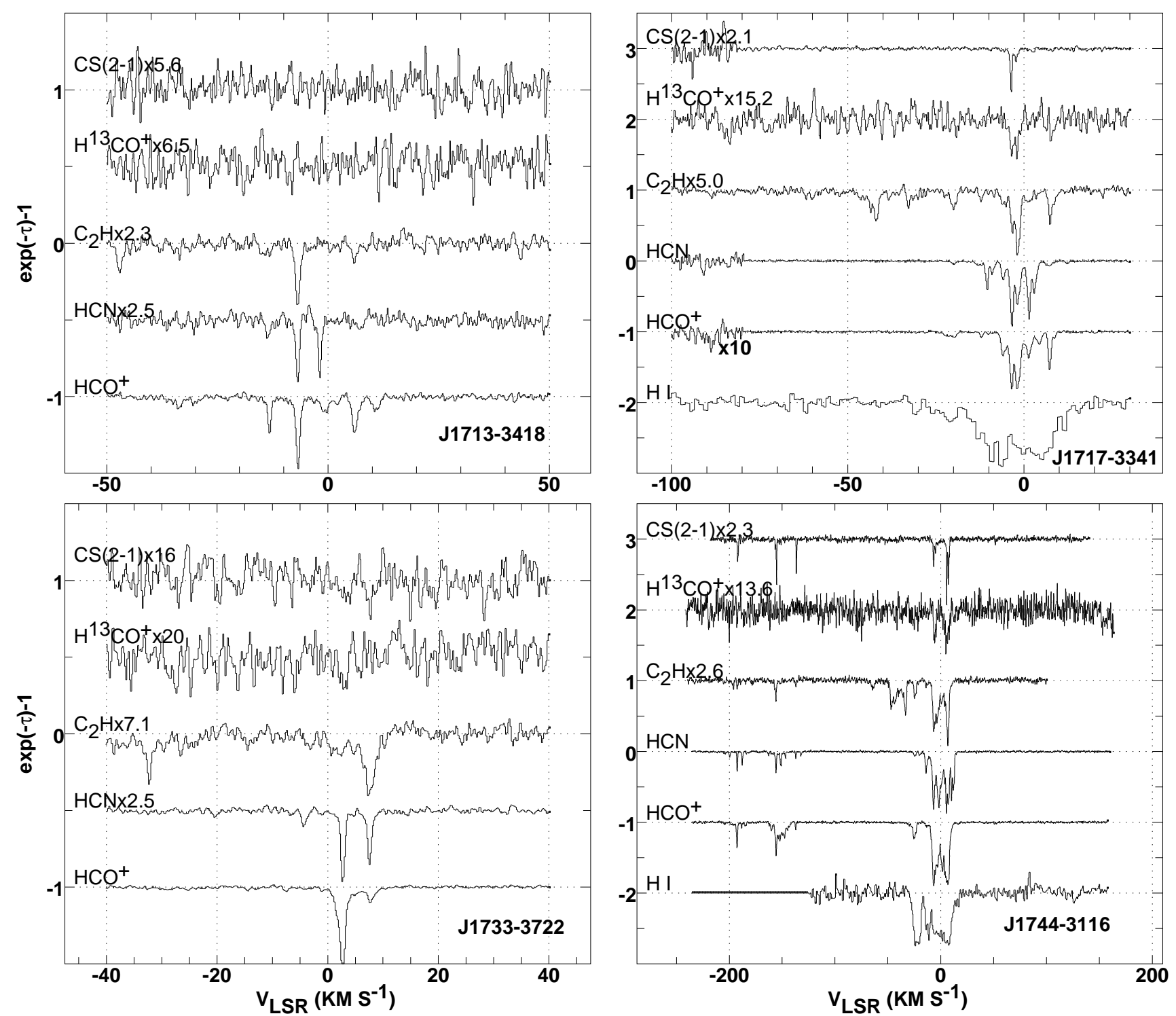

Fig. 2. ALMA absorption profiles for the four sources observed here.

an optical depth-corrected column density of atomic hydrogen as

$N(\mathrm{H} \mathrm{I})=1.823 \times 10^{18} \mathrm{~cm}^{-2} \int \frac{\tau(v) T_{\mathrm{B}}(v)}{1-\exp (-\tau(v))} \mathrm{d} v$,

where $\tau$ and $T_{\mathrm{B}}$ are the optical depth and brightness temperature and the units of velocity are $\mathrm{km} \mathrm{s}^{-1}$. In this work, $N(\mathrm{H})$ is the column density of $\mathrm{H}$-nuclei in neutral atomic and molecular form, $N(\mathrm{H})=N(\mathrm{H} \mathrm{I})+2 N\left(\mathrm{H}_{2}\right)$.

We also use the $\mathrm{CS}$ and $\mathrm{HCN}$ absorption profiles for local clouds from Lucas \& Liszt (2002) and Liszt \& Lucas (2001), respectively, and the $\mathrm{HCO}^{+}$and $\mathrm{C}_{2} \mathrm{H}$ measurements of Lucas \& Liszt (2000).

\subsection{Extinction}

We cite the $6^{\prime}$ resolution dust-emission maps scaled to optical reddening $E_{B-V}$ by Schlafly \& Finkbeiner (2011) and the 3D IR extinction map of the Galactic bulge of Schultheis et al. (2014) at the same resolution, from which we cite values of the $K$-band extinction $A_{K}$.

\section{Disk gas toward the 4 targets}

Profiles toward all sources are shown in Figs. 1-3 and some general properties of the sightlines are given in Table 1. All directions show strong molecular absorption from gas in the Galactic disk around zero velocity but only the sightline to J1744 passes inside galactocentric radius $R=1 \mathrm{kpc}$ and only that sightline shows strong molecular absorption from gas deep inside the Galactic bulge.

The sightline to J1744 is also the only one for which the gas column density is dominated by molecular gas, mostly from the disk contribution around 0-velocity. The species-by-species chemistry of this gas is not a major focus of this work, but is shown in Sect. 4 along with a variety of other data that are used as a basis of comparison with the bulge gas. Chemistry of the disk gas toward J1744 was discussed for a broader range of species observed in a low spectral resolution spectral scan by Riquelme et al. (2018), whose conclusions agree with ours regarding the similarity of the disk and bulge diffuse molecular gas components.

Although some of the molecular gas must be quite close to the Sun, for instance material in the Heeschen Cloud 


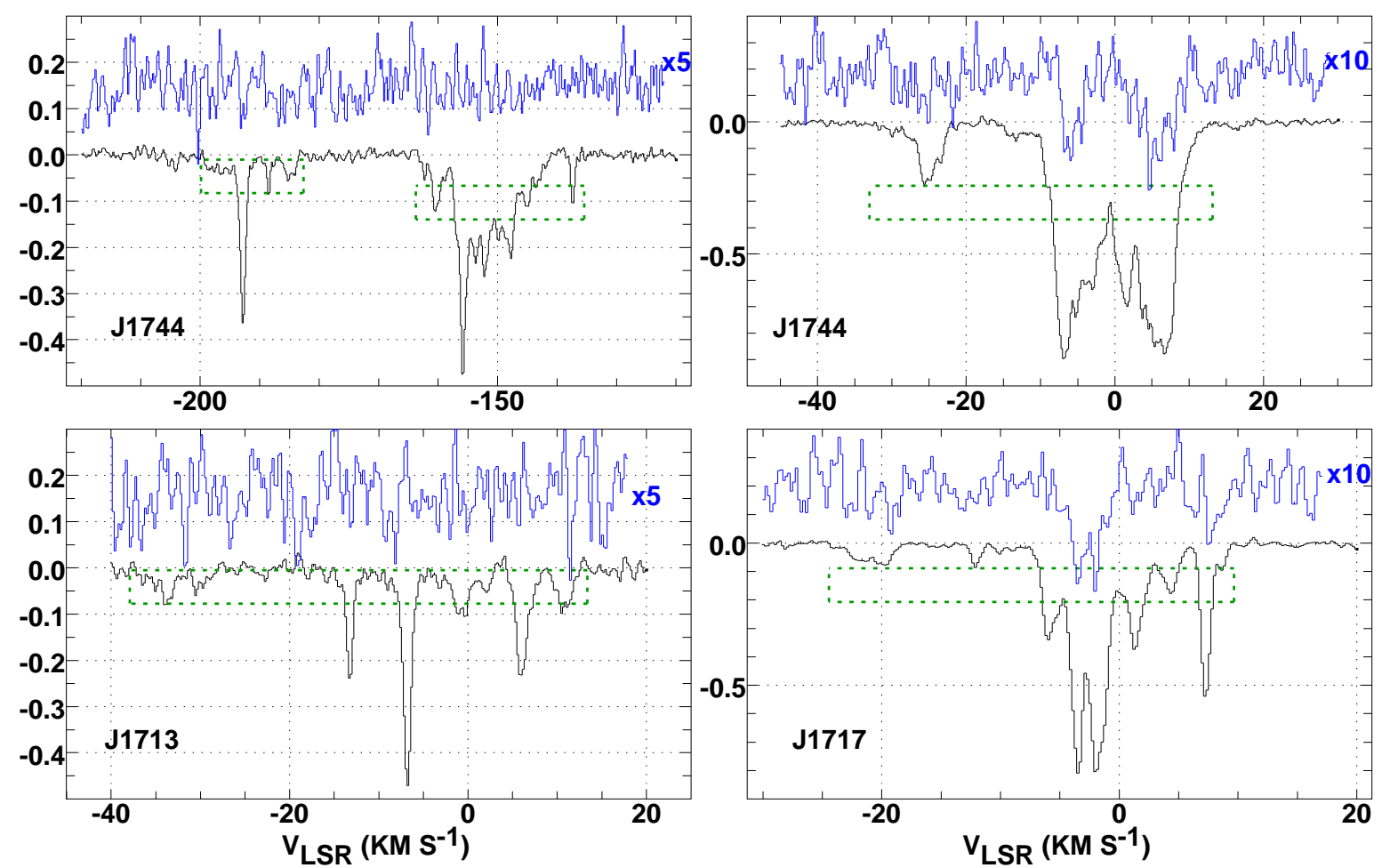

Fig. 3. $\mathrm{HCO}^{+}$and $\mathrm{H}^{13} \mathrm{CO}^{+}$absorption profiles for three of the sources observed here. The $\mathrm{H}^{13} \mathrm{CO}^{+}$are scaled upward as indicated and shown in blue over those of $\mathrm{HCO}^{+}$. Absorption toward J1744 is shown in two parts in the upper panels, with different scaling. Regions over which the profiles were integrated are shown outlined, at the mean level within each region: see Table 2 for results.

Table 2. ${ }^{12} \mathrm{C} /{ }^{13} \mathrm{C}$ isotopic abundance ratios from $\mathrm{HCO}^{+}$.

\begin{tabular}{cccccc}
\hline \hline Source & $\begin{array}{c}\text { Velocity range } \\
\mathrm{km} \mathrm{s}^{-1}\end{array}$ & $\begin{array}{c}W_{\mathrm{HCO}^{+}} \\
\mathrm{km} \mathrm{s}^{-1}\end{array}$ & $\begin{array}{c}W_{\mathrm{H}^{13} \mathrm{CO}^{+}} \\
\mathrm{km} \mathrm{s}^{-1}\end{array}$ & $\begin{array}{c}N\left(\mathrm{HCO}^{+}\right)^{a} \\
10^{12} \mathrm{~cm}^{-2}\end{array}$ & $N\left(\mathrm{HCO}^{+}\right) / N\left(\mathrm{H}^{13} \mathrm{CO}^{+}\right)^{a}$ \\
\hline $\mathrm{J} 1744$ & $-200 . .-180$ & $0.890(0.014)$ & $(0.017)$ & $0.98(0.02)$ & $>17^{b}$ \\
$\mathrm{~J} 1744$ & $-165 . .-135$ & $3.301(0.019)$ & $(0.021)$ & $3.63(0.02)$ & $>51$ \\
$\mathrm{~J} 1744$ & $-200 . .-135$ & $4.191(0.024)$ & $(0.027)$ & $4.68(0.03)$ & $>51$ \\
\hline $\mathrm{J} 1713$ & $-38 . .14$ & $2.321(0.053)$ & $(0.046)$ & $2.55(0.06)$ & $>16$ \\
$\mathrm{~J} 1717$ & $-25 . .10$ & $7.002(0.027)$ & $0.117(0.018)$ & $7.70(0.03)$ & $58 \pm 9$ \\
$\mathrm{~J} 1744$ & $-33 . .13$ & $21.852(0.056)$ & $0.333(0.023)$ & $24.03(0.06)$ & $64 \pm 4$ \\
\hline
\end{tabular}

Notes. ${ }^{(a)} N\left(\mathrm{HCO}^{+}\right)=1.10 \times 10^{12} \mathrm{~cm}^{-2} W_{\mathrm{HCO}^{+}} ; N\left(\mathrm{H}^{13} \mathrm{CO}^{+}\right)=1.135 \times 10^{12} \mathrm{~cm}^{-2} W_{\mathrm{H}^{13} \mathrm{CO}^{+}}{ }^{(b)}$ All limits are $3 \sigma$.

(Heeschen 1955) that is responsible for the H I self-absorption toward J1744, it is hard to place the disk gas in Galactic perspective. The line of sight velocity gradients due to Galactic rotation are quite shallow at the small longitudes of our sources: for a flat rotation curve with $\Theta(R)=220 \mathrm{~km} \mathrm{~s}^{-1}$, a location $5 \mathrm{kpc}$ from the Sun has $v \approx-10 \mathrm{~km} \mathrm{~s}^{-1}$ toward $\mathrm{J} 1744$ or $v \approx-40 \mathrm{~km} \mathrm{~s}^{-1}$ toward the other sources. Moreover, much of the low-velocity absorption occurs at positive velocities that are inconsistent with rotation. Positive velocities could arise from a combination of random motions and streaming associated with spiral arms, although gas at $v \gtrsim 30 \mathrm{~km} \mathrm{~s}^{-1}$ most likely arises inside the Galactic disk.

The $\mathrm{H}_{2}$ fraction falls monotonically with separation from the Galactic equator: only the line of sight toward J1744 at $b=-1^{\circ}$ has a molecular fraction exceeding 50\%. The optical depth corrections to $N(\mathrm{HI})$ in the two directions with measured $\mathrm{HI}$ absorption profiles are $35-45 \%$ of the optically thin value, but these sightlines have relatively high $\mathrm{H}_{2}$ fractions and the correction to $N(\mathrm{HI})$ is smaller than $2 N\left(\mathrm{H}_{2}\right)$.

\section{1. ${ }^{12} \mathrm{C} /{ }^{13} \mathrm{C}$ in the disk gas}

Profiles of the carbon isotopologues of $\mathrm{HCO}^{+}$are shown in Fig. 3 and results for their profile integrals and ratios of column densities are given in Table 2. Column densities are derived from integrated optical depths in the limit of no rotational excitation above the cosmic microwave background, for a molecular dipole moment of 3.92 Debye (e.g. Ando et al. 2016). From this it follows that $N\left(\mathrm{HCO}^{+}\right)=1.10 \times 10^{12} \mathrm{~cm}^{-2} \int \tau \mathrm{d} v$ and $N\left(\mathrm{H}^{13} \mathrm{CO}^{+}\right)=$ $1.135 \times 10^{12} \mathrm{~cm}^{-2} \int \tau \mathrm{d} v$ in units of $\mathrm{km} \mathrm{s}^{-1}$, accounting for the lower line frequency of the heavier isotopologue. Error estimates for the integrated optical depths in Table 2 include effects associated with the appreciable optical depths in $\mathrm{HCO}^{+}$, which were estimated using the measured rms line/continuum ratios in absorption-free regions in channel-by-channel Monte Carlo simulations of the observed profile taken as the expectation value.

We have precise measurements of the carbon $\mathrm{HCO}^{+}$isotopologues toward two sources: $N\left(\mathrm{HCO}^{+}\right) / N\left(\mathrm{H}^{13} \mathrm{CO}^{+}\right)=58 \pm 9$ toward $\mathrm{J} 1717$ and $N\left(\mathrm{HCO}^{+}\right) / N\left(\mathrm{H}^{13} \mathrm{CO}^{+}\right)=64 \pm 4$ toward $\mathrm{J} 1744$. 
This is well below the solar isotopic abundance ratio 89 , but typical for gas near the Sun observed in mm-wave absorption by Lucas \& Liszt (1998). However, some of the disk gas observed here could be situated well inside the solar circle where the ${ }^{12} \mathrm{C} /{ }^{13} \mathrm{C}$ ratio is known to be smaller (Milam et al. 2005), although still well above the very small values $20-25$ characteristic of the CMZ (Wilson 1999; Riquelme et al. 2010).

\subsection{HI spin temperature in the self-absorbed gas toward J1744}

The line of sight to J1744 is the exceedingly rare case where H I absorption (Dickey et al. 1983) has been measured in a direction where the HI emission is self-absorbed. Earlier (Gerin \& Liszt 2017) we showed that the H I self-absorption is coincident with CO $J=1-0$ emission. Here we show how the combination of $\mathrm{HI}$ absorption and self-absorption can be used to derive an upper limit on the spin temperature of the H I selfabsorbing but largely molecular gas.

We define $T_{\mathrm{B}}$ as the observed brightness temperature of the $\mathrm{HI}$ emission and $T_{\mathrm{B}, \mathrm{bg}}$ as the hypothetical brightness temperature in the absence of self-absorption: extrapolating across the self-absorption feature by Gaussian fitting of the H I emission gives $T_{\mathrm{B} \text {,bg }}=125.2 \mathrm{~K}$ in the center of the $\mathrm{HI}$ self-absorption trough where $T_{\mathrm{B}}=71.1 \mathrm{~K}$. We also define the optical depth derived from the absorption profile of Dickey et al. (1983) as the sum of foreground and background contributions $\tau=\tau_{\mathrm{fg}}+\tau_{\mathrm{bg}}$ and define $T_{\text {sp,fg }}$ and $T_{\text {sp,bg }}$ as the spin temperatures of the foreground and background emitting gases, respectively. Then the two equations

$T_{\mathrm{B}, \mathrm{bg}} \exp \left(-\tau_{\mathrm{fg}}\right)+T_{\mathrm{sp}, \mathrm{fg}}\left(1-\exp \left(-\tau_{\mathrm{fg}}\right)\right)=T_{\mathrm{B}}$

and

$T_{\mathrm{B}, \mathrm{bg}}=T_{\mathrm{sp}, \mathrm{bg}}\left(1-\exp \left(-\tau_{\mathrm{bg}}\right)\right)$

can be solved for the spin temperatures of the foreground and background gases as functions of the value of $\tau_{\mathrm{fg}}$. The minimum optical depth of the foreground gas is $\tau_{\mathrm{fg}}=0.58$ when $T_{\mathrm{sp}, \mathrm{fg}}=$ $2.73 \mathrm{~K}$. This can be compared with the total optical depth in the middle of the H I self-absorption trough, $\tau=1.22$, and it implies a fairly high minimum spin temperature for the background gas seen in emission: $\tau_{\mathrm{bg}} \leq 1.22-0.58=0.64$ and $T_{\mathrm{sp}, \mathrm{bg}} \geq 265 \mathrm{~K}$.

The implied spin temperature of the foreground selfabsorbing gas increases as $\tau_{\text {fg }}$ increases above 0.58 and the maximum optical depth, $\tau_{\mathrm{fg}}=\tau=1.22$, corresponds to $T_{\mathrm{sp}}=$ $48 \mathrm{~K}$ that is typical of gas observed in H I absorption generally (Heiles \& Troland 2003). Of course it is not realistic to assign all of the optical depth to any one component of the H I and an improved upper bound on the spin temperature of the self-absorbing gas follows from showing that the optical depth of the self-absorbing gas is below 1.22. To this end we decomposed the H I absorption profile of Dickey et al. (1983) as summarized in Table 3 and illustrated in Fig. 4. A kinematic component is found coincident with the self-absorption, having a center optical depth $\tau_{\text {fg }}=0.686 \pm 0.081$ corresponding to $T_{\text {sp,fg }}=16.2(+8.0,-10.2) \mathrm{K}$. This result unfortunately spans the range of kinetic temperatures from dark $(10 \mathrm{~K})$ to diffuse $(25 \mathrm{~K})$ molecular gas and does not allow us to decide in which regime the foreground gas exists.

The precision of the present result is limited by the noise level in the rather old absorption profile of Dickey et al. (1983). This could be improved by taking a more sensitive and contemporary H I absorption profile toward J1744, and extended by

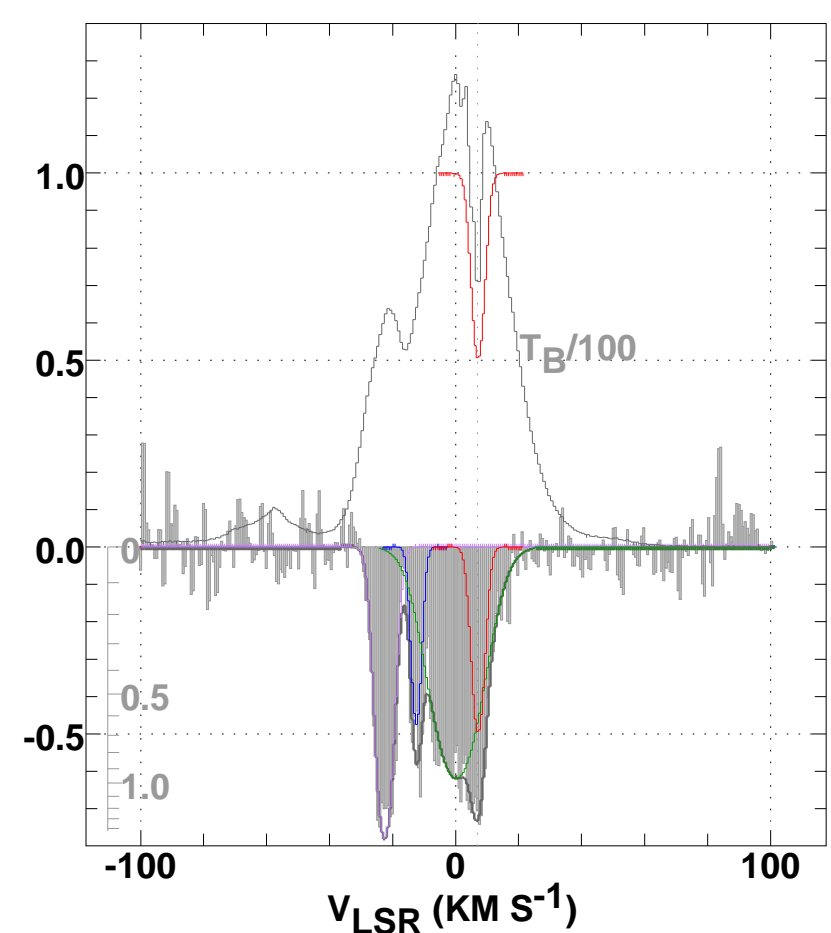

Fig. 4. $\lambda 21 \mathrm{~cm} \mathrm{HI}$ emission, absorption and self-absorption toward J1744. Bottom: Gaussian decomposition of the H I absorption profile of Dickey et al. (1983, see Table 3). An optical depth scale is shown inset. Top: GASS III H I emission scaled down by a factor 100, with one component of the Gaussian decomposition shown superposed to show coincidence between the H I absorption and self-absorption.

Table 3. Gaussian decomposition of disk H I absorption toward J1744.

\begin{tabular}{|c|c|c|c|c|}
\hline Comp & $\begin{array}{l}\text { Center } \\
\mathrm{km} \mathrm{s}^{-1}\end{array}$ & $\tau_{0}$ & $\begin{array}{c}F W H M \\
\mathrm{~km} \mathrm{~s}^{-1}\end{array}$ & $\begin{array}{c}W_{\mathrm{HI}}{ }^{a} \\
\mathrm{~km} \mathrm{~s}^{-1}\end{array}$ \\
\hline 1 & -22.602 & 1.515 & 5.876 & 9.4763 \\
\hline \pm & 0.127 & 0.109 & 0.260 & 0.515 \\
\hline 2 & -12.476 & 0.646 & 3.603 & 2.477 \\
\hline \pm & 0.154 & 0.0928 & 0.411 & 0.290 \\
\hline 3 & 0.127 & 0.967 & 17.369 & 17.874 \\
\hline \pm & 0.579 & 0.0621 & 0.957 & 0.976 \\
\hline 4 & 7.091 & 0.686 & 4.587 & 3.348 \\
\hline \pm & 0.181 & 0.081 & 0.442 & 0.330 \\
\hline
\end{tabular}

taking H I absorption profiles toward other positions with apparent H I self-absorption, which is relatively common in sightlines through the Great Rift in the inner Galaxy. Further insight into the foreground gas toward $\mathrm{J} 1744$ could also be obtained by observing ${ }^{13} \mathrm{CO}$ in emission, and by observing $\mathrm{CO}$ or ${ }^{13} \mathrm{CO}$ in absorption.

\section{Gas inside the Galactic disk}

This work shows more clearly the nuclear bulge gas at $v<$ $-135 \mathrm{~km} \mathrm{~s}^{-1}$ toward $\mathrm{J} 1744$ that was discovered in our earlier work (Gerin \& Liszt 2017; see also Riquelme et al. 2018) but otherwise we did not detect gas inside the Galactic disk except for weak features arising in the $3 \mathrm{kpc}$ arm at $-90 \mathrm{~km} \mathrm{~s}^{-1}$ toward J1717 (Fig. 1) and at $v=+52 \mathrm{~km} \mathrm{~s}^{-1}$ toward J1744. 


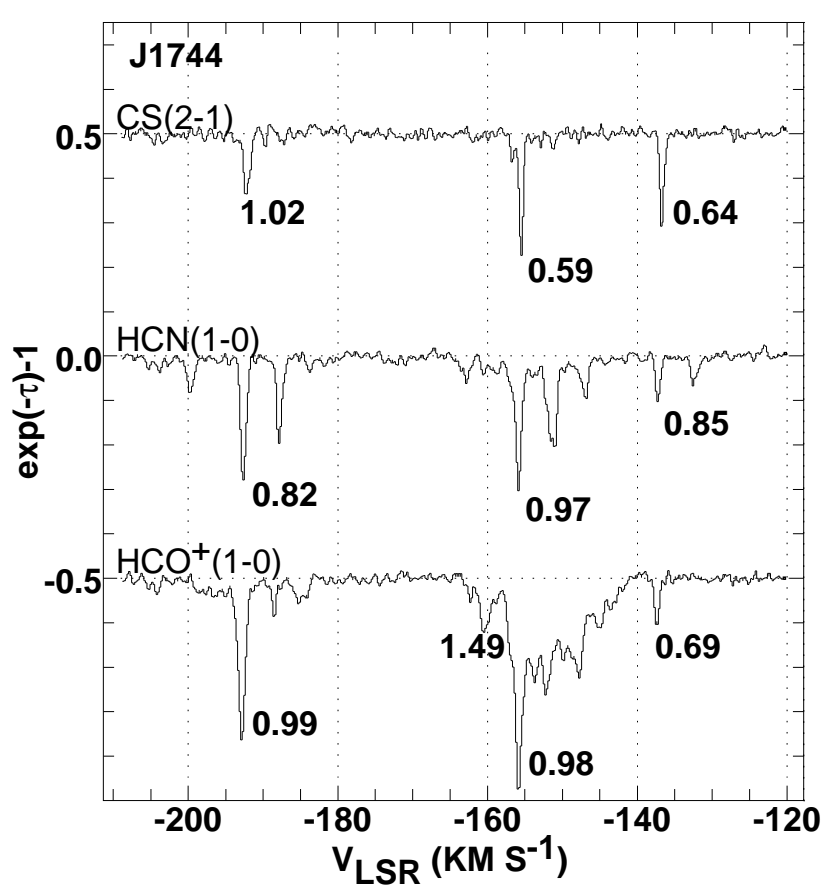

Fig. 5. $\mathrm{HCO}^{+}$, $\mathrm{HCN}$ and $\mathrm{CS}$ absorption profiles toward J1744 at velocities arising inside the Galactic bulge. The FWHM of Gaussian fits to the narrow kinematic components are indicated in units of $\mathrm{km} \mathrm{s}^{-1}$ : typical errors are $0.04 \mathrm{~km} \mathrm{~s}^{-1}$. See Tables 4,5 .

\subsection{Cloud structure and chemistry in the bulge gas toward J1744}

\subsubsection{Strucure and kinematics}

As discussed in Gerin \& Liszt (2017), especially their Table 1 and Fig. 4, gas at $v \lessgtr-135$ toward J1744 arises in the Galactic bulge at galactocentric radii $320 \mathrm{pc} \leq R_{\text {gal }} \lesssim 1500 \mathrm{pc}$ where $320 \mathrm{pc}$ is the distance of closest approach to the center and the outer radius depends on the contribution of non-circular motion to the observed velocity. A purely circular description of the motion would place all the gas at $R_{\mathrm{gal}} \lesssim 450 \mathrm{pc}$.

No matter what velocity field is adopted, the line of sight velocity gradient is very steep and the $\approx 20-25 \mathrm{~km} \mathrm{~s}^{-1}$ velocity intervals over which the molecular gas is seen, $-205 \lesssim v \lesssim$ $-185 \mathrm{~km} \mathrm{~s}^{-1}$ and $-160 \lesssim v \lesssim-135 \mathrm{~km} \mathrm{~s}^{-1}$, correspond to only $100-150 \mathrm{pc}$ along the line of sight. In each of these intervals, $\mathrm{HCO}^{+}$absorption more nearly fills the velocity range (Fig. 5), analogous to what is seen in gas near the Sun: local $\mathrm{HCO}^{+}$absorption is more ubiquitous than absorption in $\mathrm{HCN}$ or $\mathrm{CS}$ and the column densities of HCN and CS increase substantially for individual features having $N\left(\mathrm{HCO}^{+}\right) \gtrsim 10^{12} \mathrm{~cm}^{-2}$ or $W_{\mathrm{HCO}^{+}} \gtrsim 1 \mathrm{~km} \mathrm{~s}^{-1}$ (Liszt \& Lucas 2001; Lucas \& Liszt 2002).

A closer view of the absorption in the bulge gas is presented in Fig. 5. This figure shows that absorption from CS and HCN is almost entirely concentrated in narrow-lined features having profile $F W H M \lesssim 1 \mathrm{~km} \mathrm{~s}^{-1}$. $\mathrm{HCO}^{+}$also shows these (and other) narrow features, although in $\mathrm{HCO}^{+}$they are superposed over a broad and presumably more nearly volume-filling component that also appears weakly in $\mathrm{HCN}$ at $v>-160 \mathrm{~km} \mathrm{~s}^{-1}$.

The appearance of so many narrow features, and so much kinematic substructure in the broader $\mathrm{HCO}^{+}$absorption is uncharacteristic of gas observed at small galactocentric radii. It likely arises because of the unusual circumstances under which the gas is being observed. The extremely large line of sight velocity gradient can kinematically separate gas parcels that are
Table 4. Gaussian decomposition of bulge CS absorption.

\begin{tabular}{llccc}
\hline \hline Comp & $\begin{array}{l}\text { Center } \\
\mathrm{km} \mathrm{s}^{-1}\end{array}$ & $\tau_{0}$ & $\begin{array}{c}F W H M \\
\mathrm{~km} \mathrm{~s}^{-1}\end{array}$ & $\begin{array}{l}W_{\mathrm{CS}^{a}} \\
\mathrm{~km} \mathrm{~s}^{-1}\end{array}$ \\
\hline 1 & -192.6734 & 0.1440 & 1.0171 & 0.1559 \\
$( \pm)$ & 0.0184 & 0.0056 & 0.0426 & 0.0057 \\
2 & -156.6340 & 0.0633 & 0.6582 & 0.0443 \\
$( \pm)$ & 0.0332 & 0.0065 & 0.0812 & 0.0046 \\
3 & -155.5189 & 0.3153 & 0.5920 & 0.1987 \\
$( \pm)$ & 0.0072 & 0.0085 & 0.0167 & 0.0050 \\
4 & -136.7139 & 0.2349 & 0.6433 & 0.1609 \\
$( \pm)$ & 0.0094 & 0.0076 & 0.0215 & 0.0048 \\
\hline
\end{tabular}

Notes. ${ }^{(a)} N(\mathrm{CS})=8.06 \times 10^{12} \mathrm{~cm}^{-2} W_{\mathrm{CS}}$.

Table 5. Gaussian decomposition of bulge HCN absorption.

\begin{tabular}{|c|c|c|c|c|}
\hline Comp & $\begin{array}{c}\text { Center } \\
\mathrm{km} \mathrm{s}^{-1}\end{array}$ & $\tau_{0}$ & $\begin{array}{c}F W H M \\
\mathrm{~km} \mathrm{~s}^{-1}\end{array}$ & $\begin{array}{l}W_{\mathrm{HCN}}{ }^{a} \\
\mathrm{~km} \mathrm{~s}^{-1}\end{array}$ \\
\hline 1 & -192.6652 & 0.3406 & 0.8340 & 0.5480 \\
\hline$( \pm)$ & 0.0069 & 0.0037 & 0.0146 & 0.0040 \\
\hline 2 & -155.8931 & 0.2841 & 0.8530 & 0.4643 \\
\hline$( \pm)$ & 0.0089 & 0.0043 & 0.0208 & 0.0048 \\
\hline 3 & -155.5423 & 0.0297 & 8.8886 & 0.5058 \\
\hline$( \pm)$ & 0.2969 & 0.0027 & 1.4085 & 0.0331 \\
\hline 4 & -151.8292 & 0.1090 & 0.9935 & 0.2075 \\
\hline$( \pm)$ & 0.0245 & 0.0040 & 0.0616 & 0.0054 \\
\hline 5 & -137.2511 & 0.1070 & 0.8153 & 0.1672 \\
\hline$( \pm)$ & 0.0200 & 0.0046 & 0.0471 & 0.0043 \\
\hline
\end{tabular}

Notes. ${ }^{(a)} N(\mathrm{HCN})=1.89 \times 10^{12} \mathrm{~cm}^{-2} W_{\mathrm{HCN}}$.

near each other in space and have little relative motion with respect to each other. In this way, spatial substructure is revealed that would otherwise remain hidden. If seen locally the same gas distribution would be narrower in velocity and the substructure seen toward J1744 would be blended together.

\subsubsection{Chemical comparisons in the bulge gas}

To illustrate the chemistry of the bulge gas, comparing HCN and CS column densities, we decomposed the line profiles of HCN and CS into gaussian components as shown in Tables 4 and 5. To account for the HCN components that are not apparent in CS (the feature at $-151.8 \mathrm{~km} \mathrm{~s}^{-1}$ and the broad line at $-155.5 \mathrm{~km} \mathrm{~s}^{-1}$ ) we subtracted the gaussian fits in the CS profile and integrated the remainder $\mathrm{CS}$ profile over the ranges of the $\mathrm{HCN}$ components.

The results are presented in terms of HCN and CS column densities in Fig. 6 at left where we also include results for the local gas seen earlier at high Galactic latitude (Liszt \& Lucas 2001; Lucas \& Liszt 2002) and for the disk gas toward the four sightlines studied in this work. In fact, the HCN and CS column densities and their ratios in the bulge gas are very similar to those seen in local gas, with the exception of a high $\mathrm{CS} / \mathrm{HCN}$ ratio in the feature at $-137 \mathrm{~km} \mathrm{~s}^{-1}$. Comparably high $\mathrm{CS} / \mathrm{HCN}$ and $\mathrm{CS} / \mathrm{HCO}^{+}$ratios are occasionally inferred from unpublished emission measurements we have made in the vicinity of the continuum background targets used by Liszt \& Lucas (2001) and Lucas \& Liszt (2002) to study local gas in absorption. CS absorption is comparatively weak in the Galactic disk gas toward J1733, and at $v<0 \mathrm{~km} \mathrm{~s}^{-1}$ toward J1744. Similar conclusions regarding the similarity of disk and bulge gas are evident in the figures of Riquelme et al. (2018).

The hydrocarbon chemistry is illustrated at right in Fig. 6 where we compare $\mathrm{C}_{2} \mathrm{H}$ and $\mathrm{HCO}^{+}$in the new data with 
H. Liszt and M. Gerin: More on diffuse molecular gas in the Galactic bulge
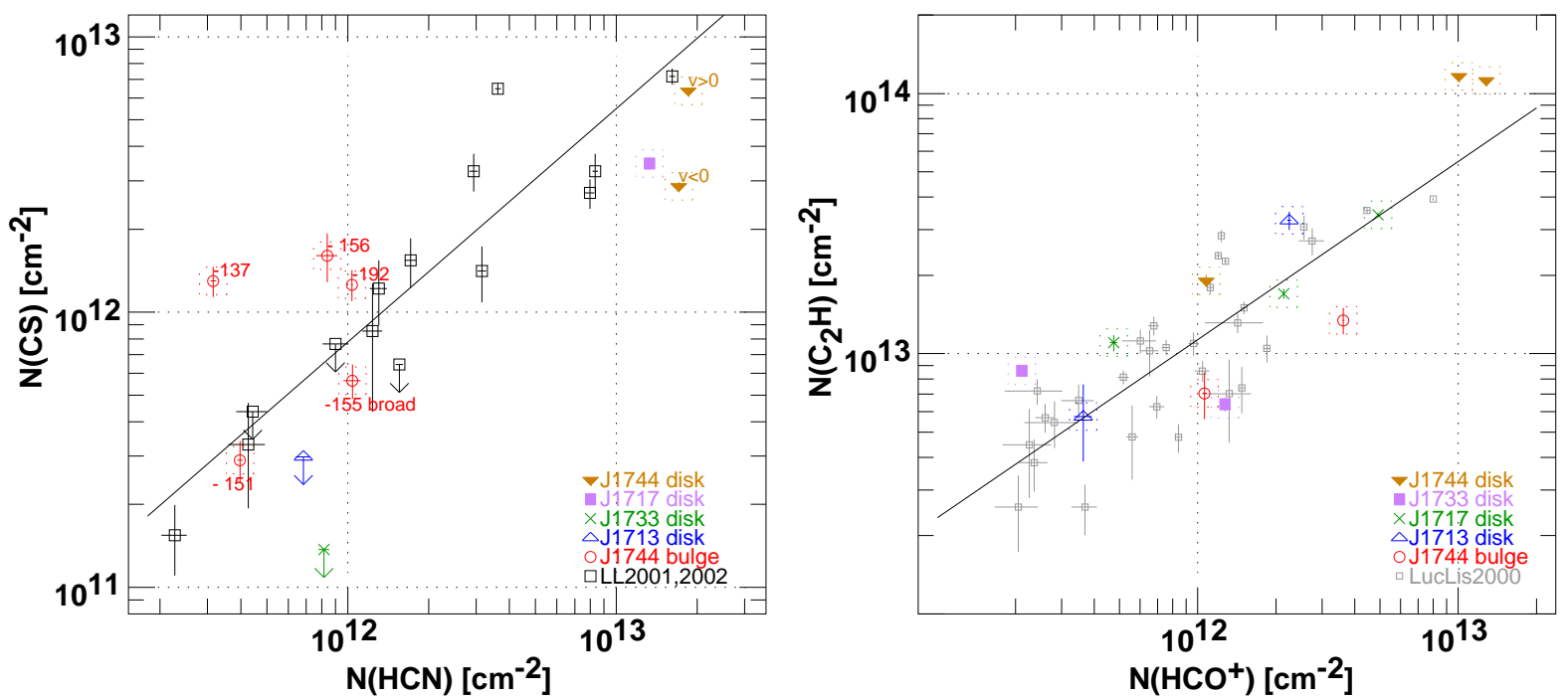

Fig. 6. Column densities for new and existing data. Left: $N(\mathrm{CS})$ vs. $N(\mathrm{HCN})$. The regression line is a power law of slope 0.85 fit to the existing data for clouds seen locally by Liszt \& Lucas (2001) and Lucas \& Liszt (2002). The velocities of features arising in the bulge toward J1744 are indicated. The disk gas toward $\mathrm{J} 1744$ is shown separately for positive and negative velocities. Right: $N\left(\mathrm{C}_{2} \mathrm{H}\right)$ vs. $N\left(\mathrm{HCO}^{+}\right)$. The regression line is a power law of slope 0.70 fit to the existing data for clouds shown in Fig. 4 of Lucas \& Liszt (2000).

that shown earlier by Lucas \& Liszt (2000). The bulge gas toward $\mathrm{J} 1744$ is at the lower edge of the range of $\mathrm{C}_{2} \mathrm{H} / \mathrm{HCO}^{+}$ abundance ratios but still within a factor two of the regression line through the older data.

\section{2. ${ }^{12} \mathrm{C} /{ }^{13} \mathrm{C}$ in the bulge gas seen toward $\mathrm{J} 1744$}

$\mathrm{HCO}^{+}$and $\mathrm{H}^{13} \mathrm{CO}^{+}$profiles are shown in more detail for three sources in Fig. 3. The profiles toward J1744 are shown in two parts corresponding to gas in the nuclear bulge at $v<$ $-135 \mathrm{~km} \mathrm{~s}^{-1}$ and Galactic disk material at $v>-40 \mathrm{~km} \mathrm{~s}^{-1}$, separately scaled. Figure 3 shows various velocity intervals over which the profiles were integrated to determine column densities, as given in Table 2.

For the gas in the nuclear bulge observed at $v<-135 \mathrm{~km} \mathrm{~s}^{-1}$ toward J1744 (Table 2) we have only a weak limit ${ }^{12} \mathrm{C} /{ }^{13} \mathrm{C}>17$ for the sub-interval $v<-190 \mathrm{~km} \mathrm{~s}^{-1}$, but ${ }^{12} \mathrm{C} /{ }^{13} \mathrm{C}>51$ overall and separately over the sub-interval $-165 \leq v \leq-135 \mathrm{~km} \mathrm{~s}^{-1}$. Apparently, this gas is not enhanced in ${ }^{13} \mathrm{C}$, which is very different from the values ${ }^{12} \mathrm{C} /{ }^{13} \mathrm{C} \approx 20-25$ seen in the CMZ (Wilson 1999; Riquelme et al. 2010). This could be disk gas that entered the bulge recently, or gas resident in the bulge that has never been astrated beyond the values characteristic of the disk in the inner Galaxy.

\subsection{Inner-Galaxy gas along the line of sight to $\mathrm{J} 1717$}

As shown in Fig. 1, HI emission from the bulge is present toward $\mathrm{J} 1717$ at $-200 \mathrm{~km} \mathrm{~s}^{-1} \lesssim v \lesssim-140 \mathrm{~km} \mathrm{~s}^{-1}$ : this was modeled as part of the tilted HI inner-galaxy gas distribution by Burton \& Liszt (1978). The H I column density $N(\mathrm{HI})=$ $4.6 \times 10^{19} \mathrm{~cm}^{-2}$ is comparable to that seen toward $\mathrm{J} 1744$ at $-165 \mathrm{~km} \mathrm{~s}^{-1} \lesssim v-135 \lesssim \mathrm{km} \mathrm{s}^{-1}, N(\mathrm{HI})=6.1 \times 10^{19} \mathrm{~cm}^{-2}$ (Gerin \& Liszt 2017), but the bulge gas toward J1717 is not dominated by $\mathrm{H}_{2}$ : the $3 \sigma$ limit on $2 \mathrm{~N}\left(\mathrm{H}_{2}\right)$ found by integrating the $\mathrm{HCO}^{+}$profile is $2 \mathrm{~N}\left(\mathrm{H}_{2}\right) \leq 5.0 \times 10^{19} \mathrm{~cm}^{-2}$.

As also shown in Fig. 1, a strong $\mathrm{HI}$ feature corresponding to the $3 \mathrm{kpc}$ arm (Bania 1977, 1980; Dame \& Thaddeus 2008) is present toward $\mathrm{J} 1717$ at $v \approx-90 \mathrm{~km} \mathrm{~s}^{-1}$. $\mathrm{HCO}^{+}$absorption
Table 6. $\mathrm{HCO}^{+}$and $\mathrm{C}_{2} \mathrm{H}$ optical depth integrals in bulge and disk gas.

\begin{tabular}{lccc}
\hline \hline Source & $\begin{array}{c}\text { Velocity range } \\
\mathrm{km} \mathrm{s}^{-1}\end{array}$ & $\begin{array}{c}W_{\mathrm{HCO}^{+}}{ }^{a} \\
\mathrm{~km} \mathrm{~s}^{-1}\end{array}$ & $\begin{array}{c}W_{\mathrm{C}_{2} \mathrm{H}}{ }^{b} \\
\mathrm{~km} \mathrm{~s}^{-1}\end{array}$ \\
\hline $\mathrm{J} 1744$ & $-210 . .-180$ & $0.964(0.018)$ & $0.108(0.022)$ \\
$\mathrm{J} 1744$ & $-165 . .-130$ & $3.296(0.019)$ & $0.206(0.024)$ \\
\hline $\mathrm{J} 1713$ & $-40 . .-22$ & $0.330(0.026)$ & $0.088(0.026)$ \\
$\mathrm{J} 1713$ & $-21 . .14$ & $2.038(0.038)$ & $0.499(0.040)$ \\
$\mathrm{J} 1717$ & $-27 . .-10$ & $0.432(0.013)$ & $0.169(0.012)$ \\
$\mathrm{J} 1717$ & $-8 . .0$ & $4.640(0.021)$ & $0.524(0.009)$ \\
$\mathrm{J} 1717$ & $0 . .1$ & $1.942(0.013)$ & $0.261(0.011)$ \\
$\mathrm{J} 1733$ & $-3 . .6$ & $1.156(0.008)$ & $0.098(0.006)$ \\
$\mathrm{J} 1733$ & $6 . .11$ & $0.592(0.005)$ & $0.132(0.005)$ \\
$\mathrm{J} 1744$ & $-31 . .-20$ & $0.978(0.015)$ & $0.292(0.016)$ \\
$\mathrm{J} 1744$ & $-16 . .0$ & $9.144(0.046)$ & $1.784(0.020)$ \\
$\mathrm{J} 1744$ & $0 . .15$ & $11.637(0.052)$ & $1.719(0.020)$ \\
\hline
\end{tabular}

Notes. ${ }^{(a)} N\left(\mathrm{HCO}^{+}\right)=1.10 \times 10^{12} \mathrm{~cm}^{-2} W_{\mathrm{HCO}^{+}} .{ }^{(b)}$ For the strongest hyperfine component only. $N\left(\mathrm{C}_{2} \mathrm{H}\right)=6.52 \times 10^{13} \mathrm{~cm}^{-2} W_{\mathrm{C}_{2} \mathrm{H}}$.

is also present toward $\mathrm{J} 1717$ over the range $-125 \mathrm{~km} \mathrm{~s}^{-1} \lesssim$ $v \lesssim-75 \mathrm{~km} \mathrm{~s}^{-1}$ but the gas is only weakly molecular: we find $N(\mathrm{HI})=2.0 \times 10^{20} \mathrm{~cm}^{-2}$ and $2 N\left(\mathrm{H}_{2}\right)=6.4 \pm 1.8 \times 10^{19} \mathrm{~cm}^{-2}$, or $f_{\mathrm{H}_{2}} \approx 0.24$.

\subsection{The far-side $3 \mathrm{kpc}$ arm toward J1744}

As seen in Fig. 1 there is a weak wing of $\mathrm{HI}$ emission at $v \gtrsim 40 \mathrm{~km} \mathrm{~s}^{-1}$ toward $\mathrm{J} 1744$, and weak $\mathrm{HCO}^{+}$absorption as well. Integrating over the velocity range $40-60 \mathrm{~km} \mathrm{~s}^{-1}$ we find $N(\mathrm{H} \mathrm{I})=8.0 \times 10^{19} \mathrm{~cm}^{-2}, 2 N\left(\mathrm{H}_{2}\right)=7.3 \pm 1.2 \times 10^{19} \mathrm{~cm}^{-2}$, i.e., the gas is $\approx 48 \%$ molecular.

\section{Summary}

Following up on the serendipitous detection of molecular absorption at velocities characteristic of the Galactic bulge toward J1744-3116 $\left(l=-2.13^{\circ}, b=-1.00^{\circ}\right)$, we observed molecular absorption toward three other quasars seen against the bulge in the fourth longitude quadrant, although at much larger 
longitude separation from the center and higher Galactic latitude (Table 1). We also reobserved the absorption toward J1744 with higher spectral resolution and broader spectral coverage, to better characterize the bulge gas. The species and transitions observed were $\mathrm{HCO}^{+}(1-0), \mathrm{HCN}(1-0), \mathrm{C}_{2} \mathrm{H}(1-0), \mathrm{CS}(2-1)$ and $\mathrm{H}^{13} \mathrm{CO}^{+}(1-0)$, all in the frequency range $87-98 \mathrm{GHz}$. Line profiles are shown in Figs. 1-3 and general properties of the sightlines are summarized in Table 1.

\subsection{Absorption arising inside the Galactic disk}

The $3 \mathrm{kpc}$ arm appeared in $\mathrm{HCO}^{+}$absorption at $-90 \mathrm{~km} \mathrm{~s}^{-1}$ toward J1717-3341 and we inferred a molecular hydrogen fraction 0.24. We also detected a weak molecular absorption feature at $+52 \mathrm{~km} \mathrm{~s}^{-1}$ toward $\mathrm{J} 1744$ from the far-side $3 \mathrm{kpc}$ arm and inferred a higher molecular fraction, 0.48, in this case. No molecular absorption was observed from the bulge gas at $-200 \mathrm{~km} \mathrm{~s}^{-1} \lesssim v \lesssim-140 \mathrm{~km} \mathrm{~s}^{-1}$ toward $\mathrm{J} 1717$, corresponding to the H I emission that was modeled as part of the tilted H I innergalaxy gas distribution by Burton \& Liszt (1978).

Absorption from CS and HCN in the bulge gas toward J1744 consists of several narrow lines with FWHM of $1 \mathrm{~km} \mathrm{~s}^{-1}$ or less (Tables 4 and 5 and Fig. 5). In $\mathrm{HCO}^{+}$the narrow lines seen in $\mathrm{CS}$ and $\mathrm{HCN}$ (and other lines not seen in $\mathrm{CS}$ or $\mathrm{HCN}$ ) are superimposed on a broader, more continuous absorption, indicating that $\mathrm{HCO}^{+}$is more widespread and more broadly distributed in space, as is the case with diffuse molecular gas observed near the Sun and more generally in the Galactic disk. The appearance of narrow lines is untypical of gas that is observed inside the Galactic disk, but the proliferation of so many features, and substructure in the $\mathrm{HCO}^{+}$profile, may be ascribed to the very large line of sight velocity gradient due to the viewing geometry. This can cause a velocity separation of gas parcels that are relatively close, and have little relative motion with respect to each other.

Comparing $\mathrm{CS}$ with $\mathrm{HCN}$ and $\mathrm{C}_{2} \mathrm{H}$ with $\mathrm{HCO}^{+}$(Fig. 6) we showed that their chemistries in the bulge gas observed toward $\mathrm{J} 1744$ at $v<-135 \mathrm{~km} \mathrm{~s}^{-1}$ resemble those seen in absorption in local and disk diffuse molecular gas. We set a limit $N\left(\mathrm{HCO}^{+}\right) / N\left(\mathrm{H}^{13} \mathrm{CO}^{+}\right)>51(3 \sigma)$ for the bulge gas toward $\mathrm{J} 1744$ (Table 2), showing that it is not enriched in ${ }^{13} \mathrm{C}$ as is material in the central molecular zone within $200 \mathrm{pc}$ of Sgr A*. The bulge gas seen toward J1744 just outside the CMZ is very clearly differentiated from gas in the $\mathrm{CMZ}$, and similar to disk gas in every aspect we examined.

\subsection{Molecular gas in the Galactic disk}

All of the sightlines showed molecular absorption from disk gas at $|v| \lesssim 40 \mathrm{~km} \mathrm{~s}^{-1}$ in $\mathrm{HCO}^{+}, \mathrm{C}_{2} \mathrm{H}$ and $\mathrm{HCN}$, and two showed absorption in disk gas from CS (2-1). Toward $\mathrm{J} 1717$ and $\mathrm{J} 1744$ we measured isotopologic abundance ratios $N\left(\mathrm{HCO}^{+}\right) / N\left(\mathrm{H}_{13 \mathrm{CO}^{+}}\right)=58 \pm 9$ and $64 \pm 4$, respectively. These are smaller than the solar abundance ratio 89 , but comparable to values seen previously in $\lambda 3 \mathrm{~mm}$ absorption from local diffuse molecular gas. Chemical abundances in the disk gas are shown in Fig. 6: they are generally like that seen previously for diffuse molecular gas seen at high Galactic latitude near the Sun, with some peculiarities: high $\mathrm{C}_{2} \mathrm{H} / \mathrm{HCO}^{+}$ratios toward $\mathrm{J} 1744$ and small $\mathrm{CS} / \mathrm{HCN}$ ratios toward $\mathrm{J} 1733$ and at $v<0 \mathrm{~km} \mathrm{~s}^{-1}$ toward J1744.

Acknowledgements. This paper makes use of the following ALMA data: ADS/JAO.ALMA\#2016.1.00132.S. ALMA is a partnership of ESO (representing its member states), NSF (USA) and NINS (Japan), together with NRC (Canada), NSC and ASIAA (Taiwan), and KASI (Republic of Korea), in cooperation with the Republic of Chile. The Joint ALMA Observatory is operated by ESO, AUI/NRAO and NAOJ. The National Radio Astronomy Observatory is a facility of the National Science Foundation operated under cooperative agreement by Associated Universities, Inc. This work was supported by the French program "Physique et Chimie du Milieu Interstellaire" (PCMI) funded by the Conseil National de la Recherche Scientifique (CNRS) and Centre National d'Etudes Spatiales (CNES). H.S.L. is grateful to the hospitality of the ITU-R, the Hotel Bel Esperance in Geneva, the JAO in Santiago and LHotel in Montreal during the completion of this manuscript. We thank Chinshin Chang at the ALMA JAO for producing the imaging scripts for this project and sheparding it through the data reduction process and we thank the referee for a close reading of the manuscript.

\section{References}

Ando, R., Kohno, K., Tamura, Y., et al. 2016, PASJ, 68, 6

Bania, T. M. 1977, ApJ, 216, 381

Bania, T. M. 1980, ApJ, 242, 95

Burton, W. B., \& Liszt, H. S. 1978, ApJ, 225, 815

Dame, T. M., \& Thaddeus, P. 2008, ApJ, 683, L143

Dickey, J. M., Kulkarni, S. R., Heiles, C. E., \& Van Gorkom, J. H. 1983, ApJS, 53, 591

Gerin, M., \& Liszt, H. 2017, A\&A, 600, A48

Heeschen, D. S. 1955, ApJ, 121, 569

Heiles, C., \& Troland, T. H. 2003, ApJ, 586, 1067

Kalberla, P. M. W., \& Haud, U. 2015, A\&A, 578, A78

Liszt, H., \& Lucas, R. 2001, A\&A, 370, 576

Lucas, R., \& Liszt, H. 1998, A\&A, 337, 246

Lucas, R., \& Liszt, H. S. 2000, A\&A, 358, 1069

Lucas, R., \& Liszt, H. S. 2002, A\&A, 384, 1054

Milam, S. N., Savage, C., Brewster, M. A., Ziurys, L. M., \& Wyckoff, S. 2005, ApJ, 634, 1126

Morris, M., \& Serabyn, E. 1996, ARA\&A, 34, 645

Riquelme, D., Amo-Baladrón, M. A., Martín-Pintado, J., et al. 2010, A\&A, 523, A51

Riquelme, D., Bronfman, L., Mauersberger, R., et al. 2018, A\&A, 610, A43

Schlafly, E. F., \& Finkbeiner, D. P. 2011, ApJ, 737, 103

Schultheis, M., Chen, B. Q., Jiang, B. W., et al. 2014, A\&A, 566, A120

Wilson, T. L. 1999, Rep. Progr. Phys., 62, 143 